\title{
Diferenças na qualidade de vida e insegurança alimentar entre homens e mulheres vivendo com HIV/Aids no estado da Paraíba, Brasil
}

\author{
Differences in quality of life and food insecurity between men \\ and women living with HIV/AIDS in the state of Paraíba, Brazil
}

Rafaela Lira Formiga Cavalcanti de Lima (https://orcid.org/0000-0003-1272-0067) ${ }^{1}$

Mickella de Farias Silva (https://orcid.org/0000-0002-0044-394X) ${ }^{1}$

Nadjeanny Ingrid Galdino Gomes (https://orcid.org/0000-0002-6367-2176) ${ }^{1}$

Jeffrey Nickollas Cavalcanti da Silva (https://orcid.org/0000-0002-5601-7130) ${ }^{1}$

Maria Augusta Correa Barroso Magno Viana (https://orcid.org/0000-0002-1122-1197) ${ }^{1}$

Rodrigo Pinheiro de Toledo Vianna (https://orcid.org/0000-0002-5358-1967) ${ }^{1}$

${ }^{1}$ Universidade Federal da Paraíba. Jardim Universitário s/n, Castelo Branco. 58051-900 João Pessoa PB Brasil. rafaelanutri@gmail.com

\begin{abstract}
A prevalence study was conducted to compare quality of life and food insecurity in men and women living with HIV/AIDS. The sample comprised $481 \mathrm{HIV}$-infected individuals undergoing antiretroviral therapy at a referral hospital in the State of Paraiba, Brazil. Food insecurity and quality of life were assessed using the Brazilian Household Food Insecurity Scale and WHOQOL-HIV Bref, respectively. The results were presented as absolute and relative frequencies and gender differences were tested using the chi-squared test adopting a significance level of 0.05 . The findings showed that $40.1 \%$ of the sample were women. A higher percentage of women than men had a low income and low education level (65.8\% and $72.5 \%$, respectively). Prevalence of food security was lower in women than in men $(29.0 \%$ compared to $42.7 \%)$, and a higher percentage of women than men reported below average quality of life (54.9\% compared to $44.4 \%)$. The findings reveal that, besides the usual difficulties faced by $H I V$-infected patients, this group showed a significant level of gender inequality. The management of HIV patient care should consider these important findings, promoting access to care and support services and gender equality so that women can live fairer and more equal lives.

Key words HIV, Gender Identity, Food and Nutritional Security, Quality of Life
\end{abstract}

Resumo Estudo de prevalência realizado com pessoas vivendo com HIV/Aids, em terapia antirretroviral, com o objetivo de comparar a qualidade de vida e a insegurança alimentar entre homens e mulheres. Foram incluidos aleatoriamente quatrocentos e oitenta e um indivíduos que buscaram atendimento hospitalar. A insegurança alimentar foi avaliada pela Escala Brasileira de Insegurança Alimentar e a qualidade de vida pelo instrumento WHOQOL-HIV-Bref. Os resultados foram apresentados em frequência absoluta e relativa $e$ as diferenças entre os sexos foram testadas com o teste qui-quadrado, considerando significância de 5\%. Do total da amostra, 40,1\% eram do sexo feminino e essas apresentaram piores condições de renda (65,8\%), baixa escolaridade (72,5\%), menor prevalência de segurança alimentar (29,02\%) e qualidade de vida abaixo da média (54,9\%), comparadas com os homens $(44,4 \%)$. Os resultados do estudo mostram que além das dificuldades enfrentadas pelos portadores do vírus HIV, este grupo apresenta uma importante desigualdade de gênero e a gestão do cuidado voltado a estas pessoas deve considerar este importante achado, promovendo o acesso a politicas sociais e promovendo a isonomia entre os gêneros, em prol de uma vida feminina mais justa e igualitária.

Palavras-chave HIV, Identidade de Gênero, Segurança Alimentar e Nutricional, Qualidade de vida 


\section{Introdução}

Antes da introdução da terapia antirretroviral (TARV), na metade da década de 1990, pessoas infectadas com o vírus HIV progrediam rapidamente para a Aids e consequentemente à morte. Nos dias atuais o tratamento adequado permite uma expectativa de vida quase igual à de uma pessoa não infectada ${ }^{1}$. Em 2017, havia 36,9 milhões [31,1 milhões - 43,9 milhões] de pessoas vivendo com HIV no mundo. Embora seja mais prevalente entre os homens todas as semanas cerca de 7.000 mulheres jovens entre 15 e 24 anos são infectadas pelo HIV. Globalmente, o HIV é a principal causa de morte entre mulheres de 30 a 49 anos $^{2}$. No Brasil, desde o início da epidemia até junho de 2018, foram notificados $926.742 \mathrm{mil}$ casos de infecção pelo vírus HIV e até 31 de dezembro de 2017, foram notificados 327.655 óbitos tendo o HIV/Aids como causa básica ${ }^{3}$.

Pessoas vivendo com HIV/Aids (PVHA) se configuram em um grupo específico e vulnerável $^{4}$ que se encontra em tratamento permanente, servindo-se de uma terapêutica complexa e de alto custo. As Diretrizes Terapêuticas para Manejo da Infecção pelo HIV em adultos no Brasil destacam a importância da adesão ao tratamento para o controle da doença, sendo importante a realização de alimentação saudável para o bom funcionamento do organismo, preservar o sistema imunológico, melhorar a tolerância aos antirretrovirais, tanto favorecendo sua absorção como prevenindo os efeitos colaterais ${ }^{5,6}$.

Com o aumento da sobrevida devido à TARV, as pessoas infectadas pelo vírus da imunodeficiência humana passaram a ter preocupações não só com a capacidade do tratamento de aumentar seus anos de vida, mas também com a manutenção de sua qualidade de vida ${ }^{7}$. A qualidade de vida é uma necessidade básica importante e está relacionada também com o aumento ou redução da morbimortalidade da população ${ }^{8}$. Estudos demonstram uma correlação positiva entre melhor qualidade de vida e a adesão à TARV. Esta, por sua vez, influencia diretamente a contagem de carga viral e está associada ao aparecimento e ao estágio da doença e seus sintomas?.

Da mesma forma, estudos apontam que a Insegurança Alimentar e Nutricional se associa a uma pior adesão ao tratamento em pessoas vivendo com HIV/Aids ${ }^{10}$. A insegurança alimentar e nutricional é uma inequívoca violação do direito humano a uma alimentação adequada, ocasionando a irregularidade do acesso a alimentos de qualidade, em quantidade suficiente para a manutenção de uma vida saudável, de forma socialmente aceitável respeitando também as características culturais dos indivíduos ${ }^{11}$.

O aumento da prevalência de mulheres infectadas pelo vírus HIV é uma realidade e por isso, é importante conhecer se as condições de vida dessas é semelhante àquelas vivenciadas pelos homens para um melhor manejo da doença.

A questão que se procurou responder foi se existe diferença entre as prevalências de insegurança alimentar e nos escores de qualidade de vida entre os sexos de pessoas vivendo com HIV/ Aids, por se tratar de indicadores que estão relacionados com a adesão ao tratamento: condição fundamental para o não aparecimento ou o controle dos sintomas da doença Aids.

\section{Métodos}

Foi realizado um estudo transversal com uma amostra de pessoas vivendo com HIV/Aids em acompanhamento no Complexo Hospitalar de Doenças Infectocontagiosas Clementino Fraga (CHCF) em João Pessoa-PB, hospital de referência estadual para acompanhamento e diagnóstico dos casos de HIV.

Todas as pessoas que compareceram ao serviço para acompanhamento clínico, durante o período de 2 de setembro até 23 de dezembro de 2015 foram elegíveis para ser incluídas no estudo. Este período foi independente de qualquer planejamento do serviço, compreendendo as atividades de rotina do Hospital.

Os critérios de inclusão no estudo foram ter infecção comprovada pelo HIV, em qualquer estágio (assintomático, sintomático e/ou Aids), ter mais que 18 anos e estar em TARV há mais de seis meses, dentre os medicamentos mais utilizados estão os inibidores de transcriptase reversa análogos de nucleotídeos e os inibidores de protease. Foram excluídos aqueles que apresentassem algum estado clínico que impedisse a participação na pesquisa, indivíduos em situação de confinamento ou institucionalizados e gestantes. No período determinado para coleta de dados, todas as 503 PVHA que atendiam os critérios de inclusão foram recrutadas e $22(4,4 \%)$ foram excluídas por incompletude das respostas ao instrumento de coleta de dados, geralmente por serem chamadas para consulta médica e não retornarem para entrevista após a conclusão da mesma, logo, a amostra final do estudo foi composta de 481 pessoas.

Entrevistadores previamente treinados realizaram entrevistas face a face no momento em 
que os indivíduos aguardavam a sua consulta médica. $\mathrm{O}$ instrumento de coleta de dados com as informações analisadas neste trabalho contemplou os tópicos apresentados no Quadro 1.

O WHOQOL-HIV-Bref, escala de medida utilizada neste estudo para avaliar a qualidade de vida, instrumento composto por 31 itens, com escala de pontuação de 1 a 5 e divididos em 6 domínios, amplamente utilizado em diversos países. Possui bom desempenho psicométrico e alta confiabilidade ( 27 itens apresentaram Alfa de Conbach maior que 0,70 e os demais valores entre: 0,32 a 0,65$)$, apresentando excelente consistência interna e validade para as facetas e domínios que aborda e é especialmente recomendada para estudos com PVHA ${ }^{12,13}$. Neste trabalho considerou-se somente a pontuação total da escala, segundo os critérios de pontuação da $\mathrm{WHO}^{12}$.

A Escala Brasileira de Insegurança Alimentar é um instrumento adaptado da escala americana Household Food Security Survey Module (HFSSM) e validado por Segall-Corrêa et al. ${ }^{14}$, em 2003 com ampla utilização em estudos epidemiológicos no Brasil.

Trata-se de uma escala de 14 itens ou somente 8 itens, quando a família não tem nenhum integrante menor de 18 anos, que classifica as famílias em situação de segurança ou em diferentes níveis de insegurança alimentar, desde insegurança leva, passando por moderada e insegurança grave, a pior das situações, quando é observada a ocorrência de fome entre os integrantes da família, de acordo com a somatória das respostas positivas dos itens.

Todos os dados foram duplamente digitados em planilha eletrônica e o banco de dados foi analisado com o auxílio do programa estatístico Stata SE, versão 14. As variáveis foram descritas em tabelas de frequências e para verificar a relação entre duas variáveis categóricas, foi utilizado o teste de associação Qui-quadrado de Pearson adotando-se o nível de significância de 5\% para rejeitar a hipótese de não associação.

A pesquisa seguiu as diretrizes éticas contempladas na Resolução 466/2012, do Conselho Nacional da Saúde ${ }^{15}$. Todos os participantes aceitaram participar da pesquisa assinando o TCLE, em duas vias, uma direcionada ao participante e outra ao pesquisador. A pesquisa foi aprovada pelo Comitê de Ética em Pesquisa do Centro de Ciências da Saúde da Universidade Federal da Paraíba.

\section{Resultados}

Do total de pessoas incluídas na pesquisa, 40,1\% eram do sexo feminino. As características da amostra, estratificada por sexo são mostradas na Tabela 1 .

Os participantes apresentaram idade mínima de 18 e máxima de 87 anos de idade com mediana de 44 anos, idade escolhida para categorizar a amostra em dois grupos iguais no que diz respeito à faixa etária. Os homens apresentaram uma frequência maior de escolaridade fundamental completa ou superior que as mulheres, assim como maior renda per capita, 54,9\% dos homens vs. $34,2 \%$ das mulheres tinha renda per capita média acima de meio salário mínimo. Conviver com companheiro, ou ter alguma ocupação foram características semelhantes entre homens e mulheres.

Quadro 1. Tópicos contidos no instrumento de coleta de dados e suas respectivas descrições. João Pessoa-PB, 2015.

\begin{tabular}{|l|l|}
\hline \multicolumn{1}{|c|}{ Tópicos } & \multicolumn{1}{c|}{ Descrição } \\
\hline $\begin{array}{l}\text { Caracterização do } \\
\text { paciente }\end{array}$ & $\begin{array}{l}\text { Informações sócio demográficas: número de prontuário, data de nascimento, idade, } \\
\text { sexo, escolaridade, estado civil, renda familiar (valor ou número de salários mínimos), } \\
\text { ocupação e contagem de residentes, por sexo e idade no domicilio. }\end{array}$ \\
\hline $\begin{array}{l}\text { Sobre o histórico do } \\
\text { HIV/Aids }\end{array}$ & $\begin{array}{l}\text { Ano do primeiro teste positivo, ano em que acha que foi infectado, como acha que foi } \\
\text { infectado, se faz uso de medicamentos e em que ano começou a tomá-los, como está a } \\
\text { sua saúde, qual é o seu estágio de HIV e se considera estar doente atualmente. }\end{array}$ \\
\hline Sobre os hábitos & $\begin{array}{l}\text { Hábitos de fumar, beber e usar drogas (atuais e passados), de realizar atividades } \\
\text { físicas por mais de trinta minutos, três vezes por semana e a descrição destas em caso } \\
\text { afirmativo. }\end{array}$ \\
\hline Qualidade de vida & Escala WHOQOl-HIV Bref. \\
\hline $\begin{array}{l}\text { Avaliação da } \\
\text { insegurança alimentar }\end{array}$ & Escala Brasileira de Insegurança Alimentar - EBIA. \\
\hline
\end{tabular}

Fonte: Elaborado pelos autores. 
Com relação à qualidade de vida, o sexo feminino apresentou uma frequência maior que $o$ masculino de pessoas com medidas de qualidade de vida abaixo da média $(54,9 \%$ vs. $44,4 \%)$ (Tabela 2).

A prevalência de insegurança alimentar e nutricional encontrada neste grupo foi de $62,8 \%$, com 18,0\% em seu nível de maior gravidade (Figura 1).

Entretanto quando esta característica foi analisada estratificando-se por sexo, observou-se que as mulheres apresentavam situação pior que os homens, com menor frequência de segurança alimentar e maiores frequência de insegurança alimentar nos níveis mais graves, insegurança moderada $(24,9 \%$ entre as mulheres vs. $12,8 \%$ entre os homens) e insegurança grave $(19,2 \%$ entre as mulheres vs. $16,7 \%$ entre os homens), é importante ressaltar que existe associação entre a insegurança alimentar e qualidade de vida em ambos os sexos (Tabela 3). Entre as mulheres à medida que a segurança alimentar se agrava, maior é a prevalência de qualidade de vida abaixo da média, entre as seguras $(28,6 \%)$ e entre as com insegurança alimentar grave $(75,7 \%)$. Entre os homens a maior prevalência é encontrada entre os que apresenta IA moderada $(78,4 \%)$.

Tabela 1. Diferenças entre as frequências das variáveis sociodemográficas e econômicas com o sexo em PVHA. João Pessoa-PB, 2015.

\begin{tabular}{|c|c|c|c|c|}
\hline \multirow[b]{2}{*}{ Variáveis } & \multicolumn{2}{|c|}{ Sexo } & \multirow{2}{*}{$\begin{array}{c}\text { Total } \\
\text { n (\%) }\end{array}$} & \multirow[b]{2}{*}{ p-valor ${ }^{\star}$} \\
\hline & $\begin{array}{c}\text { Feminino } \\
\mathbf{n}(\%)\end{array}$ & $\begin{array}{c}\text { Masculino } \\
\text { n (\%) }\end{array}$ & & \\
\hline Idade & & & & 0,425 \\
\hline Até 44 anos & $100(51,8)$ & $153(53,1)$ & $253(52,6)$ & \\
\hline Mais de 44 anos & $93(48,2)$ & $135(46,9)$ & $228(47,4)$ & \\
\hline Escolaridade & & & & 0,006 \\
\hline Até Fundamental Incompleto & $140(72,5)$ & $176(61,5)$ & $316(65,7)$ & \\
\hline Fundamental Completo ou mais & $53(27,5)$ & $112(38,9)$ & $165(34,3)$ & \\
\hline Estado Civil & & & & 0,291 \\
\hline Convive com companheiro & $71(36,8)$ & $97(33,9)$ & $168(35,1)$ & \\
\hline Não convive com companheiro & $122(63,2)$ & $189(66,1)$ & $311(64,9)$ & \\
\hline Renda Per capita & & & & 0,000 \\
\hline Até 1 ² salário mínimo & $127(65,8)$ & $130(45,1)$ & $257(53,4)$ & \\
\hline Mais que $1 / 2$ salário mínimo & $66(34,2)$ & $158(54,9)$ & $224(46,6)$ & \\
\hline Ocupação & & & & 0,380 \\
\hline Com ocupação/Ativo & $90(46,6)$ & $129(44,8)$ & $219(45,5)$ & \\
\hline Sem ocupação/Inativo & $103(53,4)$ & $159(55,2)$ & $262(54,5)$ & \\
\hline Total & $193(100)$ & $288(100)$ & $481(100)$ & \\
\hline
\end{tabular}

*Teste qui-quadrado de Pearson.

Fonte: Elaborado pelos autores.

Tabela 2. Diferenças entre a avaliação da qualidade de vida média com o sexo em PVHA, João Pessoa-PB, 2015.

\begin{tabular}{llccc}
\hline \multirow{2}{*}{ Avaliação Qualidade de Vida Média } & \multicolumn{3}{c}{ Sexo } & Total \\
\cline { 2 - 4 } & \multicolumn{1}{c}{$\begin{array}{c}\text { Feminino } \\
\mathbf{n}(\%)\end{array}$} & $\begin{array}{c}\text { Masculino } \\
\mathbf{n}(\%)\end{array}$ & n (\%) & p-valor $^{*}$ \\
\hline Abaixo da média & $106(54,9)$ & $128(44,4)$ & $234(48,6)$ & 0,015 \\
Acima da média & $87(45,1)$ & $160(55,6)$ & $247(51,4)$ & \\
Total & $193(100)$ & $288(100)$ & $481(100)$ & \\
\hline
\end{tabular}

${ }^{\star}$ Teste qui-quadrado de Pearson.

Fonte: Elaborado pelos autores. 


\section{Discussão}

Os resultados deste trabalho mostraram, como muitos estudos já vêm revelando, a feminilização da infecção pelo vírus HIV e consequentemente a mudança do perfil da população atingida ${ }^{1,3,16}$. Quase metade dos pacientes acompanhados, realizando tratamento com TARV, no hospital de referência do Estado da Paraíba, o Complexo

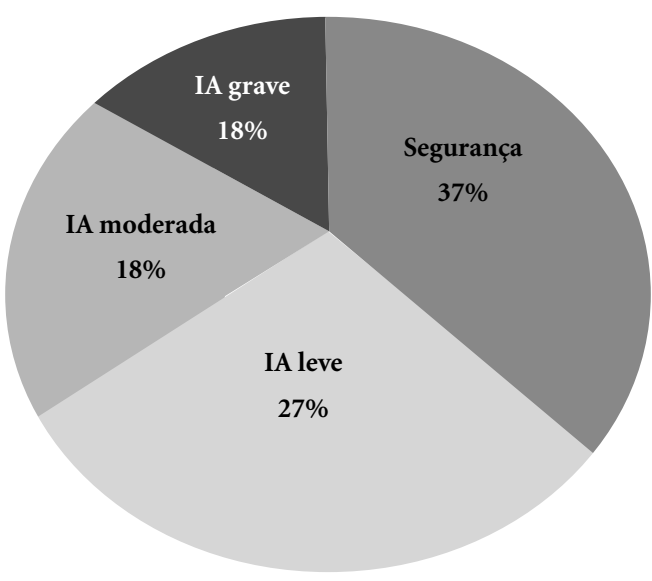

Figura 1. Níveis de insegurança alimentar nutricional em PVHA. João Pessoa-PB, 2015.

Fonte: Elaborado pelos autores.
Hospitalar Clementino Fraga, são mulheres. Este fato tem implicações diretas na gestão do cuidado destes indivíduos e especialmente considerando os papeis sociais que os diferentes gêneros têm na sociedade ${ }^{17}$, assim como o significado disto na própria sociedade machista e patriarcal onde as mulheres ocupam uma posição desprivilegiada.

A desigualdade feminina, como visto, não é uma questão meramente sociológica, mas também de saúde e nutricional, cabendo à sociedade como um todo o dever de lutar pela isonomia entre os gêneros, em prol de uma vida feminina mais justa e igualitária ${ }^{18}$.

Outra característica importante é o número crescente de pessoas mais velhas acometidas pela infecção. A mediana da idade dos pacientes deste estudo foi em torno de 44 anos, confirmando o que estudos tem mostrado com relação ao aumento da suscetibilidade da infecção em todas as faixas etárias, porém com uma preocupação maior com o aumento no número de casos em idosos, outra mudança no perfil da doença ${ }^{19,20}$. Em uma pesquisa realizada em um hospital geral na cidade de Montevidéu, Uruguai, em 2013 com 198 indivíduos foi identificado que os pacientes com HIV admitidos para internação, tinham idade média de 63 anos e uma porcentagem similar de ambos os sexos, homens $53 \%$ e $47 \%$ de mulheres ${ }^{21}$.

O perfil dos pacientes em acompanhamento no CHCF, com relação a viver com companheiro, foi semelhante entre os sexos e confirmou os resultados mostrados em outros estudos, como o

Tabela 3. Diferenças entre a avaliação da qualidade de vida média com os níveis de insegurança alimentar, segundo sexo em PVHA, João Pessoa-PB, 2015.

\begin{tabular}{|c|c|c|c|c|}
\hline \multirow[b]{2}{*}{ Insegurança Alimentar } & \multicolumn{2}{|c|}{ Avaliação qualidade de vida média } & \multirow[b]{2}{*}{$\begin{array}{l}\text { Total } \\
\text { n }(\%)\end{array}$} & \multirow[b]{2}{*}{ p-valor ${ }^{*}$} \\
\hline & $\begin{array}{c}\text { Abaixo da média } \\
\text { n (\%) }\end{array}$ & $\begin{array}{c}\text { Acima da média } \\
\mathbf{n}(\%)\end{array}$ & & \\
\hline Homens & & & & 0,000 \\
\hline Segurança & $32(26,0)$ & $91(74,0)$ & $123(100)$ & \\
\hline IA Leve & $40(50,0)$ & $40(50,0)$ & $80(100)$ & \\
\hline IA Moderada & $29(78,4)$ & $8(21,6)$ & $37(100)$ & \\
\hline IA Grave & $27(56,3)$ & $21(43,8)$ & $48(100)$ & \\
\hline Total & $128(44,4)$ & $160(55,6)$ & $288(100)$ & \\
\hline Mulheres & & & & 0,000 \\
\hline Segurança & $16(28,6)$ & $40(71,4)$ & $56(100)$ & \\
\hline IA Leve & $27(51,9)$ & $25(48,1)$ & $52(100)$ & \\
\hline IA Moderada & $35(72,9)$ & $13(27,1)$ & $48(100)$ & \\
\hline IA Grave & $28(75,7)$ & $9(24,3)$ & $37(100)$ & \\
\hline Total & $106(54,9)$ & $87(45,1)$ & $193(100)$ & \\
\hline
\end{tabular}

^Teste qui-quadrado de Pearson.

Fonte: Elaborado pelos autores. 
de Tesfaye et al..$^{22}$ realizado na Etiópia que observou apenas $37,3 \%$ das pessoas infectadas casadas. Neste estudo, apesar de apresentar percentual semelhante, não foram observadas as características das relações, o que pode ser diferente entre os gêneros e também com relação a presença de crianças no domicílio, ou seja, famílias completas ou não. Estudo de validação da EBIA para PVHA mostrou que a composição das famílias era diferente quando a pessoa infectada era do gênero masculino ou do feminino, sendo mais comum a presença de filhos no segundo grupo ${ }^{5}$. É de se esperar que a presença de filhos possa alterar os cuidados de adesão à TARV, porém, não existem estudos avaliando a composição familiar com a adesão ao tratamento em PVHA.

Além das diferenças de gênero em decorrência da cultura patriarcal hegemônica, outras diferenças agravam ainda mais a situação das mulheres, como as menores escolaridades e menores rendas per capita observadas entre as mulheres deste estudo, dado semelhante ao encontrado em estudo realizado em seis cidades brasileiras que entrevistou com profundidade oitenta e cinco mulheres, entre 18 e 49 anos, vivendo com HIV/ Aids cuja análise do perfil das entrevistadas mostrou um quadro de vulnerabilidade social, expresso pelo baixo nível de escolaridade, precária inserção no mercado de trabalho e presença de violência na vida ${ }^{23}$.

Esta situação não é nenhum privilégio da realidade brasileira, uma vez que em Cuba, pesquisa realizada em uma policlínica com pacientes infectados pelo HIV, em 2015, foi observado que entre pacientes do sexo feminino prevaleceu o nível primário de ensino e no sexo masculino dominou o nível secundário ${ }^{24}$. A diferença da renda per capta, onde a maior proporção de mulheres está na faixa de até $1 / 2$ salário mínimo, em comparação com os homens, é outro problema importante para a gestão do cuidado deste grupo populacional.

Sendo as PVHA um grupo vulnerável biologicamente, o percentual de pessoas que se encontram em insegurança alimentar é alarmante, totalizando $62,8 \%$ de inseguros o que representa o dobro da prevalência da média da população brasileira $^{25}$. Em países mais desenvolvidos, a realidade não é diferente, em estudos publicados nos anos de 2005, 2011 e 2013 a partir dos dados de uma coorte de indivíduos infectados pelo HIV em TARV altamente ativa na Columbia Britânica no Canadá os seguintes resultados foram encontrados: a ocorrência de insegurança alimentar foi quase 5 vezes maior do que na população geral canadense; uma alta (71\%) prevalência de insegurança alimentar entre indivíduos que recebem TARV e que a insegurança alimentar está associada a um compêndio de fatores ambientais e comportamentais; e em grupos extremamente vulneráveis, como os usuários de drogas injetáveis e que recebem TARV com insegurança alimentar a probabilidade de morte foi duas vezes maior do que a daqueles que apresentavam segurança alimentar ${ }^{26-28}$.

Pior situação é observada em nossos resultados quando a insegurança alimentar foi estratificada por sexo. Nas situações mais graves, quando há redução da quantidade de alimento no domicílio (insegurança moderada) ou mesmo a ocorrência de fome entre os moradores (insegurança grave) a prevalência destes eventos é maior entre as mulheres, em comparação com os homens. Sendo as mulheres, na cultura brasileira, as principais responsáveis pela administração dos recursos domésticos e preparação da alimentação da família, este resultado não mostra uma contradição, mas uma situação muito grave que estas pessoas e suas famílias enfrentam no seu cotidiano.

Estudos realizados da Uganda em 2013 e 2014 mostram que 902 PVHA em uso de TARV que estavam em insegurança alimentar, tiveram maior chance de uma evolução clínica insatisfatória, contribuindo para uma maior mortalidade e piores desfechos clínicos na amostra acompanhada. $\mathrm{O}$ acesso a alimentos e a qualidade da dieta estão associados à qualidade de vida e devem ser considerados como parte das intervenções destinadas a mitigar as consequências psicossociais do HIV ${ }^{10,29}$. Os dados do presente estudo demonstram associação entre a qualidade de vida e a insegurança alimentar em ambos os sexos, com dados mais preocupantes em relação às mulheres uma vez que a qualidade de vida dessas se mostra pior do que a dos homens.

Pessoas que vivem sem acesso adequado aos alimentos, vivenciam constrangimentos que em PVHA podem vir a ser piores devido à discriminação e à demanda de outras necessidades terapêuticas ${ }^{30}$. Atingir e manter uma nutrição ideal é considerada um complemento importante no atendimento clínico de pacientes infectados pelo HIV, pois uma boa nutrição pode melhorar a função imunológica de um indivíduo, limitar as complicações específicas da doença e melhorar a qualidade de vida e a sobrevivência ${ }^{6}$.

Com relação à qualidade de vida, também chamou a atenção o fato de os resultados deste trabalho mostrarem que as mulheres percebem sua situação pior sobre si mesmas do que perce- 
bem os homens. Na Etiópia, em 2016, pesquisadores também observaram disparidades de gênero ao avaliar 1.180 pessoas vivendo com HIV/ Aids usuários da TARV quanto à qualidade de vida relatada, impacto social, econômico e psicológico. A partir de uma análise multivariada de seus dados os autores observaram que, a angústia psicológica, a baixa contagem de CD4, o desemprego e a insegurança alimentar e nutricional foram associados a menores escores de qualidade de vida em ambos os sexos, entretanto nas mulheres, os efeitos da insegurança na qualidade de vida foram maiores, o que foi explicado pelo fato de as mulheres desempenharem um papel maior nas responsabilidades domésticas nessa região ${ }^{31}$.

A igualdade de gênero e a não discriminação são direitos humanos fundamentais e componentes de um mundo saudável, pacífico e próspero. A igualdade de gênero tem estado na agenda global da saúde há décadas, no entanto, a desigualdade de gênero continua a persistir em todo o mundo. Essa, juntamente com a discriminação prejudicam o progresso na resposta à Aids e privam mulheres e meninas de direitos básicos e sua capacidade de prevenir o HIV e de acessar os serviços que precisam quando são portadoras do vírus ${ }^{32,33}$. A igualdade de gênero e o empoderamento feminino são pontos essenciais para acabar com a epidemia de Aids até $2030^{2}$.

Estes resultados em conjunto formam um quadro negativo da situação das mulheres infectadas com o vírus HIV em que um ciclo complexo e inter-relacionado de fatores pode potenciar os efeitos negativos de cada fator. A restrição de renda, menor escolaridade, insegurança alimentar e menor qualidade de vida, concomitantemente, podem fazer com que este grupo se torne mais vulnerável, e em se tratando de mulheres, soma-se ainda as desigualdades de gênero vivenciadas na cultura nacional.

\section{Conclusão}

Apesar dos avanços quanto a sobrevida de pessoas vivendo com HIV/Aids nos últimos tempos, ainda se mostram desafiadoras as dimensões que envolvem a gestão do cuidado e a qualidade de vida dessas pessoas.

Este estudo mostrou que, no Estado da $\mathrm{Pa}$ raíba, as PVHA em acompanhamento clínico no hospital de referência, além de serem biologicamente mais vulneráveis, também são socialmente vulneráveis devido à baixa escolaridade, baixa renda per capta, falta de ocupação e famílias incompletas.

Este estudo de prevalência traz informações novas e relevantes na medida que mostra que entre pacientes vivendo com HIV/Aids em tratamento com antirretrovirais a situação das mulheres é pior que a dos homens uma vez que elas apresentam maior restrição de renda, menor escolaridade, prevalências mais elevadas de insegurança alimentar, especialmente nos níveis mais graves e pior qualidade de vida.

Sabe-se que a população feminina historicamente sofre com desigualdades, o presente estudo comprova esse reflexo da sociedade nas PVHA no estado da Paraíba e que a desigualdade feminina não é apenas uma questão sociológica, mas também de saúde, qualidade de vida e nutricional.

Compete à rede de cuidado voltada para as PHVA considerar estes aspectos, no sentido de promover um ciclo positivo de enfrentamento do HIV/Aids além da questão biológica, viabilizando o acesso a políticas sociais e favorecendo a isonomia entre os gêneros, em prol de uma vida feminina mais justa e igualitária. 


\section{Colaboradores}

RLFC Lima, MF Silva e RPT Vianna e trabalharam na concepção, delineamento, análise e interpretação dos dados, redação do artigo e aprovação da versão final. NIG Gomes, JNC Silva e MACBM Viana trabalharam na pesquisa e metodologia da versão final.

\section{Referências}

1. Programa das Nações Unidas no combate à Aids (UNAIDS). Estatísticas globais sobre HIV 2017 [Internet]. 2018 [acessado 2019 mar 20]. Disponível em: https://unaids.org.br/estatisticas/.

2. Programa das Nações Unidas no combate à Aids (UNAIDS). Acelerando o progresso rumo à igualdade de gênero nas Nações Unidas e além [Internet]. 2018 [acessado 2019 mar 20]. Disponível em: https:// unaids.org.br/2018/04/acelerando-o-progresso-emdirecao-igualdade-de-genero-nas-nacoes-unidas-e -alem/.

3. Brasil. Ministério da Saúde (MS). Boletim epidemiológico HIV/Aids 2018. Brasília: MS; 2018.

4. Jesus GJ, Oliveira LB, Caliari JS, Queiroz AAFL, Gir E, Reis RK. Dificuldades do viver com HIV/Aids: Entraves na qualidade de vida. Acta Paul Enferm 2017; 30(3):301-307.

5. Medeiros ARC, Lima RLFC, Medeiros LB, Trajano FMP, Salerno AAP, Moraes RM, Vianna RPT. Insegurança alimentar moderada e grave em famílias integradas por pessoas vivendo com HIV/Aids: validação da escala e fatores associados. Cien Saude Colet 2017; 22(10):3353-3364.

6. Grobler L, Siegfried N, Visser ME, Mahlungulu SSN, Volmink J. Nutritional interventions for reducing morbidity and mortality in people with HIV. Cochrane Database Syst Rev 2013; 2:CD004536.

7. O'Connell KA, Skevington SM. An International Quality of Life Instrument to Assess Wellbeing in Adults Who are HIV-Positive: A Short Form of the WHOQOL-HIV (31 items). AIDS Behav 2012; 16(2):452-460.

8. Guimarães MDC, Carneiro M, Abreu DMX, França EB. HIV/AIDS Mortality in Brazil, 2000-2015: Are there reasons for concern? Rev Bras Epidemiol 2017; 20(Supl. 1):182-190.

9. Geocze L, Mucci S, De Marco MA, Nogueira-Martins LA, Citero VA. Quality of life and adherence to HAART in HIV-infected patients. Rev Saude Publica 2010; 44(4):743-749.

10. Palermo T, Rawat R, Weiser SD, Kadiyala S. Food Access and Diet Quality Are Associated with Quality of Life Outcomes among HIV-Infected Individuals in Uganda. PLoS ONE 2013; 8(4):e62353.

11. Brasil. Lei no 1.346 , de 15 de setembro de 2016. Cria o Sistema Nacional de Segurança Alimentar e Nutricional - SISAN com vistas em assegurar o direito humano à alimentação adequada e dá outras providências. Diário Oficial da União 2006; 18 set.

12. Whoqol Hiv Group. WHOQOL-HIV for quality of life assessment among people living with HIV and AIDS: results from the field test. AIDS Care 2004; 16(7):882-889.

13. Zimpel RR, Fleck MP. Quality of life in HIV-positive Brazilians: application and validation of the WHOQOL-HIV, Brazilian version. AIDS Care 2007; 19(7):923-930.

14. Segall-Corrêa AM, Escamilla RP, Maranha LK, Sampaio MFA. (In) Segurança Alimentar no Brasil: Validação de metodologia para acompanhamento e avaliação. Campinas: UNICAMP; 2003. 
15. Brasil. Ministério da Saúde (MS). Conselho Nacional de Saúde (CNS). Resolução no 466, de 12 de dezembro de 2012. Diário Oficial da União 2013; 13 dez.

16. Hipolito RL, Oliveira DC, Costa TL, Marques SC, Pereira ER, Gomes AMT. Quality of life of people living with HIV/AIDS: temporal, socio-demographic and perceived health relationship. Rev Lat Am Enferm 2017; 25:e2874.

17. Oliveira FBM, Queiroz AAFLN, Sousa ÁFL, Moura MEB, Reis RK. Sexual orientation and quality of life of people living with HIV/Aids. Rev Bras Enferm 2017; 70(5):1004-1010.

18. Lima ACO, Lima RSV, Silva JMA. Gênero feminino, contexto histórico e segurança alimentar. DEMETRA Aliment Nutr Saude 2016; 11:789-802.

19. Santos AFM, Assis M. Vulnerability of the elderly to HIV/AIDS: public politics and health professionals in the context of integral care: a literature review. Rev Bras Geriatr Gerontol 2011; 14(1):147-157.

20. Torres TS, Cardoso SW, Velasque LS, Marins LMS, Oliveira MS, Veloso VG, Grinsztejn B. Aging with HIV: an overview of an urban cohort in Rio de Janeiro (Brazil) across decades of life. Braz J Infect Dis 2013; 17(3):324-331.

21. Silva AG, Cavalcanti VS, Santos TS, Bragagnollo GR, Santos KS, Santos IMS, Mousinho KC, Fortuna CM. Revisão integrativa da literatura: assistência de enfermagem a pessoa idosa com HIV. Rev Bras Enferm 2018; 71(Supl. 2):884-892.

22. Tesfaye M, Kaestel P, Olsen MF, Girma T, Yilma D, Abdissa A, Ritz C, Prince M, Friis H, Hanlon C. Food insecurity, mental health and quality of life among people living with HIV commencing antiretroviral treatment in Ethiopia: a cross-sectional study. Health Qual Life Outcomes 2016; 14:37.

23. Villela WV, Barbosa RM. Trajetórias de mulheres vivendo com HIV/aids no Brasil. Avanços e permanências da resposta à epidemia. Cien Saude Colet 2017; 22(1):87-96.

24. Estrada JAF, Hechavarría OB, Pullés Fernández MC, Tabares L, Fong JO. Percepción de riesgo de sida en adultos mayores de un área de salud. MEDISAN 2015; 19(9):1115-1120.

25. Instituto Brasileiro de Geografia e Estatística (IBGE). Pesquisa Nacional por Amostra de Domicilios - Segurança Alimentar 2013. Ministério do Planejamento Orçamento e Gestão. Rio de Janeiro: IBGE; 2013.

26. Normén L, Chan K, Braitstein P, Anema A, Bondy G, Montaner JSG, Hogg RS. Food insecurity and hunger are prevalent among HIV-positive individuals in British Columbia, Canada. J Nutr 2005; 135(4):820-825.

27. Anema A, Weiser SD, Fernandes KA, Ding E, Brandson EK, Palmer A, Montaner JSG, Hogg RS. High prevalence of food insecurity among HIV-infected individuals receiving HAART in a resource-rich setting. AIDS Care 2011; 23(2):221-230.

28. Anema A, Chan K, Chen Y, Weiser S, Montaner JSG, Hogg RS. Relationship between Food Insecurity and Mortality among HIV-Positive Injection Drug Users Receiving Antiretroviral Therapy in British Columbia, Canada. PLoS ONE 2013; 8(5):e61277.
29. Rawat R, Faust E, Maluccio JA, Kadiyala S. The Impact of a Food Assistance Program on Nutritional Status, Disease Progression, and Food Security Among People Living With HIV in Uganda: JAIDS J Acquir Immune Defic Syndr 2014; 66(1):e15-e22.

30. Programa das Nações Unidas no combate à Aids (UNAIDS). UNAIDS Policy Brief: HIV, Food Security and Nutrition [Internet]. 2008 [acessado $2015 \mathrm{fev}$ 10]. Disponível em: http://www.tandfonline.com/doi/ abs/10.2989/AJAR.2009.8.4.4.

31. Vo QT, Hoffman S, Nash D, El-Sadr WM, Tymejczyk OA, Gadisa T, Melaku Z, Kulkarni SG, Remien RH, Elul B. Gender Differences and Psychosocial Factors Associated with Qualityof Life Among ART Initiators in Oromia, Ethiopia. AIDS Behav 2016; 20(8):16821691.

32. Programa das Nações Unidas no combate à Aids (UNAIDS). Agir para mudar leis discriminatórias [Internet]. 2019 [acessado 2019 mar 10]. Disponível em: https://unaids.org.br/wp-content/uploads/2019/02/ ZeroDiscrimina\%C3\%A7\%C3\%A3o2019_Brochura. pdf.

33. Programa das Nações Unidas no combate à Aids (UNAIDS). UNAIDS Gender Action Plan 2018-2023 - A framework for accountability [Internet]. 2018 [acessado 2019 mar 10]. Disponível em: https://www. unaids.org/sites/default/files/media_asset/jc2925_ unaids-gender-action-plan-2018-2023_en.pdf.

Artigo apresentado em 03/05/2019

Aprovado em 01/04/2020

Versão final apresentada em 03/04/2020

Editores-chefes: Romeu Gomes, Antônio Augusto Moura da Silva 
